\title{
Modified FOLFOX6 Regimen
}

National Cancer Institute

\section{Source}

National Cancer Institute. Modified FOLFOX6 Regimen. NCI Thesaurus. Code C155746.

A chemotherapy regimen consisting of leucovorin, infusional fluorouracil and oxaliplatin used for the treatment of advanced-stage and metastatic colorectal cancer. This modified version differs from the FOLFOX6 regimen in that the dose of oxaliplatin is lower. 